\section{Measurement and Analysis of Multiband Bistatic and Monostatic Radar Signatures of Wind Turbines}

\author{
M. Ritchie, F. Fioranelli, A. Balleri and H.D. Griffiths
}

This paper presents the results of recent measurements taken with two radar systems to measure the simultaneous monostatic and bistatic signature of wind turbines, at S-band and X-band. Coherent monostatic and bistatic data was collected with the University College London (UCL) NetRAD 2.4 GHz radar, and the Cranfield University CW radar operating at X-band. This initial analysis shows the bistatic Doppler signature of wind turbines and informs on the key differences seen at modest bistatic angles. Polarimetric variations are also analysed via data gathered using co-polarised $\mathrm{VV}$ and $\mathrm{HH}$ and cross-polarised $\mathrm{VH}$ components.

Introduction: The United Kingdom, together with many other countries, is investing a large amount of money to support the development of alternative and sustainable ecologically-friendly technologies, including wind farms, as a means to reduce reliance on fossil fuels and generation of greenhouse gases. The United Kingdom has targeted that $15 \%$ of energy should be generated by renewable sources by 2020 [1]. The EU committee has also recognised that wind power will be the dominant technology to achieve this target because wind farms are the most mature technology currently available [1].

Radar systems detect and localise targets by sending an electromagnetic signal and detecting target echoes. If the target is moving, the frequency of its echo is shifted by a quantity that is proportional to the target velocity.

Radar systems are used by Air Traffic Control (ATC) air defence networks to provide services to aircraft and to detect and localise threats and hostile air targets. It has long been recognised that wind turbines can significantly disrupt the operation of surveillance radars used for air traffic control and for air defence. The radar return from wind farms, commonly called wind farm clutter, presents time-varying amplitude levels that can be comparable to those produced by aircraft. Depending on weather conditions, the rotating blades can have very high tip speeds and induce similar Doppler shifts to those of aircraft. Wind farms cause detection problems to radar systems both because of the increased clutter level and because of the obscuration of true targets behind the wind farm. This represents a potentially significant safety risk to the services provided to aircraft and for the reliability of air defence. Currently, half of wind farm developments in the UK face objections from aviation stakeholders and the $\mathrm{MoD}$ on the grounds of radar interference and obstruction or impact to low flying operations [2].

Significant research has been applied into analysing the effects of wind turbines on radar systems [3-4]. Research that focused on mitigating the issues caused by wind turbines includes new wind turbine designs [5], holographic radar techniques [6] as well as additional digital signal processing algorithms [7].

The application of bistatic or multistatic radar systems as a solution to wind farm clutter interference is investigated here. The aim of these measurements was to provide the first result of simultaneous monostatic and bistatic Doppler from an operational wind turbine.

Bistatic radars provide additional degrees of freedom which can result in a number of advantages against the wind farm signals, such as 1) lower radar target/clutter cross sections 2) lower Doppler spreads and 3) multi-perspective information.

There potentially exists an optimal geometry that reduces the level and Doppler spread of wind farm clutter whilst keeping air target returns high, hence less wind farm interference. Additionally, the bistatic geometry allows the antenna beams to be directed so to minimise obstructions. A bistatic configuration provides more information and can result in a significantly reduced probability of interference. Very little real data has been published on multistatic radar wind farm clutter making this area of research exceptionally novel.

Radar systems and measurements: The experiments were carried out in January 2015 at the Westmill Wind Farm in Watchfield, outside the perimeter of the Defence Academy of the UK. The wind farm consists of 5 turbines which are $49 \mathrm{~m}$ in height and have blades with a length of $31 \mathrm{~m}$. During the measurements the average wind velocity was recorded as approximately $4 \mathrm{~ms}-1$.

Two separate radar systems were used to generate the results presented in this letter. One was the NetRAD S-band multistatic pulsed coherent radar system developed at UCL [8]. This system used $200 \mathrm{~mW}$ transmit power, a pulse length of $0.6 \mu \mathrm{s}, 45 \mathrm{MHz}$ bandwidth and a PRF of $5 \mathrm{kHz}$ during the experiments. The antennas used had beamwidth of $10^{\circ}$ and a gain of $24 \mathrm{dBi}$. The second system is the Cranfield University $\mathrm{X}$-band $\mathrm{CW}$ radar. This radar used a transmit power of $15 \mathrm{dBm}$ and was centred at about $10 \mathrm{GHz}$. Two NetRAD nodes were used during the experiments to allow simultaneous monostatic and bistatic recordings. The X-band CW radar was simultaneously deployed at the monostatic node of the NetRAD radar to give comparative X-band data.

The geometry of the setup can be seen in Fig. 1. These nodes were separated by a distance of $50 \mathrm{~m}$ on a baseline that was $432 \mathrm{~m}$ from the 2nd turbine, hereafter labelled the Turbine under Test (TUT), giving a bistatic angle of approximately $6.6^{\circ}$. All five turbines were visible to the radar but only Doppler data from the TUT is shown from the NetRAD radar.

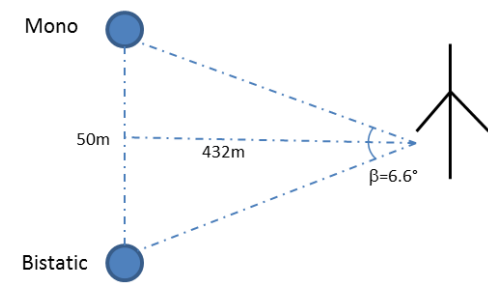

Fig. 1 Plan view of the experimental setup

Data analysis: The data was processed to provide both Range Time Intensity (RTI) and Doppler-Time spectrogram from the turbines. The key comparisons made here are between the simultaneous monostatic and bistatic datasets. Fig. 2 shows the RTI plot of the turbines as seen from the NetRAD monostatic radar. This figure shows the history of 5 seconds of pulse compressed data. The 5 turbines are the vertical spaced lines at the two way range distances from the radar.
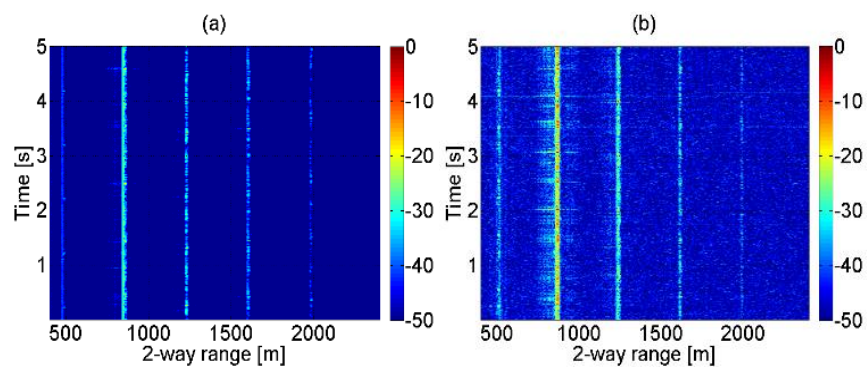

Fig. 2 NetRAD HH Polarised RTI (a) Monostatic and (b) Bistatic

The following analysis shows the Doppler signatures generated by the TUT. In order to produce the Doppler signature a Short Time Fourier Transform (STFT) was used with a weighted Hamming window of $0.6 \mathrm{~s}$ duration and an overlap of $95 \%$. The Doppler-Time spectrogram from both the monostatic and bistatic node are shown in Fig. 3. Clear differences can be observed between the two spectrograms. The monostatic Doppler spread is much higher, and shows a symmetrical pattern, whereas the bistatic Doppler is dominated by a negative Doppler component.

The antennas were then rotated to capture bistatic VV polarised data. This is shown in Fig. 4. In this case the monostatic Doppler is again shown to have a higher return, but the bistatic signature is less asymmetrical in comparison to the HH dataset. For completeness the cross polarised results are shown in Fig. 5. As expected these results show a much suppressed return, with some blade flashes seen in the monostatic data but very little returns from the blades in the bistatic node. 
It was observed that for both the TUT and the 3rd turbine, the positive Doppler blade flashes have higher intensity than those with negative Doppler when using VV data. This effect seems to be more evident in the monostatic signatures. In the $\mathrm{HH}$ data it is seen that the negative Doppler blade flashes become more intense than the positive ones. This was particularly evident with the TUT $\left(\beta=6.5^{\circ}\right)$ and less so for the 3 rd turbine $\left(\beta=4.65^{\circ}\right)$. The ratio of the blade flash intensity to the central nacelle Doppler component was found to be greater (1-2 dBs) in all cases for the monostatic data compared to bistatic result. Although only a small difference was found in this data it may become more significant at greater bistatic angles. Further bistatic experiments are required to validate this relationship fully, particularly for greater bistatic angles.
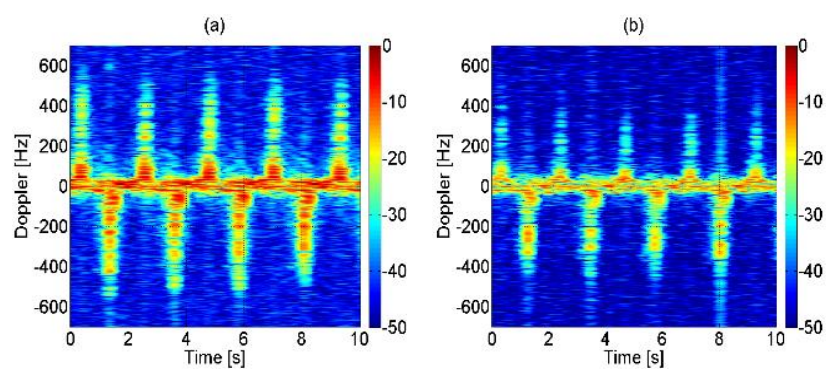

Fig. 3 NetRAD HH Polarised (a) Monostatic and (b) Bistatic spectrogram
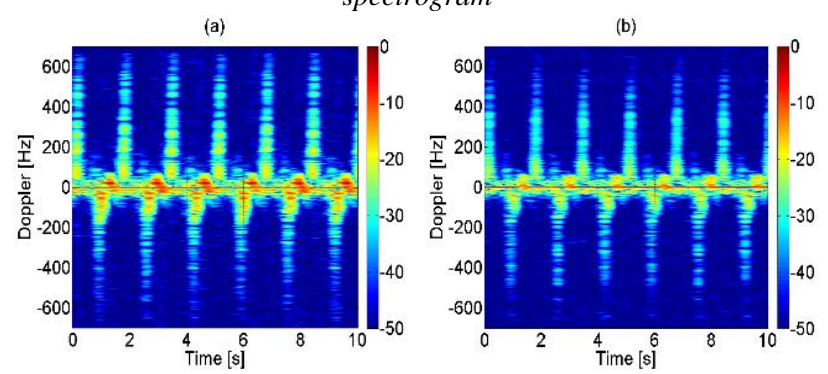

Fig. 4 NetRAD VV Polarised (a) Monostatic and (b) Bistatic spectrogram
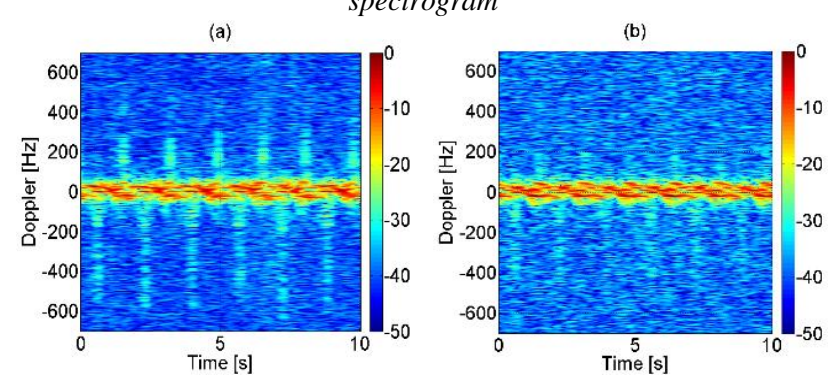

Fig. 5 NetRAD VH Polarised (a) Monostatic and (b) Bistatic spectrogram

The X-band radar results can be seen within Fig. 6. The results are related back to the $\mathrm{S}$-band figures such that the monostatic results in Fig. 3-5 are equivalent to Fig. $6 a, 6 b \& 6 c$ respectively. Unlike the Sband radar the $\mathrm{CW} \mathrm{X}$-band system does not resolve targets in range so all of the turbines signatures have been folded into the Doppler spectrogram shown. This leads to multiple non-periodic blade flashes, seen particularly in Fig. $6 a$.

The X-band data results show a higher Doppler return within the positive Doppler component of the signature compared to the negative component in both $\mathrm{HH}$ and VV. This does not correspond with the mono and bistatic $\mathrm{HH}$ returns seen in the S-band data, where a stronger negative component is observed. Both bands of data do show a stronger relative return in $\mathrm{HH}$ pol data as well as a wider blade flash component in time compared to VV data.

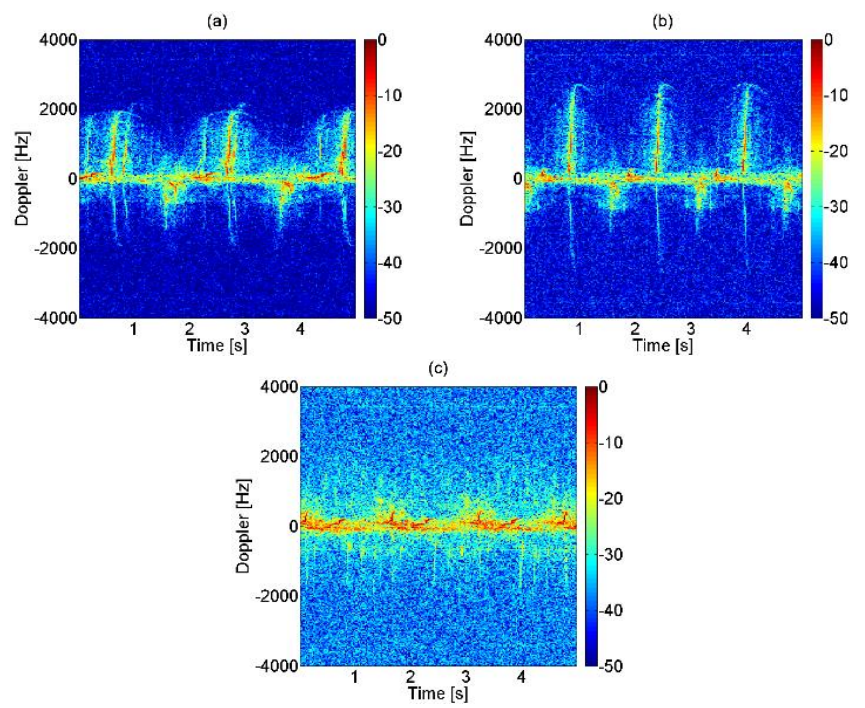

Fig. $6 \mathrm{X}$-Band Mono spectrogram (a) HH Pol (b) VV pol (c) VH Pol

Conclusion: In this letter experimental results from a simultaneous $\mathrm{S}$ band monostatic and bistatic have been shown, along with complementary X-band monostatic data. The key differences between the monostatic and bistatic Doppler spectra have been described and how these translate to performance of a bistatic or multistatic system when dealing with wind turbine clutter. These results are believed to represent the first publication of simultaneous coherent monostatic and bistatic Doppler from wind turbines. Further experimentation is required to investigate these variations as a function of bistatic angle (particularly wider bistatic angles) and different geometries.

Acknowledgments: This work was funded partially by the IET A F Harvey Prize awarded to Prof Hugh Griffiths (2013).

M. Ritchie, F. Fioranelli and H. Griffiths (Electronic and Electrical Engineering, UCL, Torrington Place, London, United Kingdom, WC1E $7 J E)$

E-mail: m.ritchie@ucl.ac.uk

A. Balleri (Centre for Electronic Warfare, Cranfield University, Defence Academy of the United Kingdom, SN6 8LA)

\section{References}

1. "The EU's Target for Renewable Energy: 20\% by 2020", Volume I: Report, Authority of the House of Lords, 24 Oct. 2008

2. http://www.renewableuk.com/en/our-work/aviation-andradar/index.cfm, Accessed 6/3/15.

3. Y.F. Lok, A. Palevsky, and J. Wang, Simulation of radar signal on wind turbine, IEEE Aerospace and Electronic Systems Magazine, 26(8):39-42, August 2011.

4. A. Naqvi, Shang-Te Yang, and Hao Ling, Investigation of doppler features from wind turbine scattering, IEEE Antennas and Wireless Propagation Letters, 9:485-488, May 2010.

5. A. Balleri, A. Al-Armaghany, H.D. Griffiths, K.F. Tong, T. Matsuura, T. Karasudani and Y. Ohya, "Measurements and analysis of the radar signature of a new wind turbine design at $\mathrm{X}$ band", IET Radar Sonar \& Navigation, vol. 7, no. 2, pp. 170-177, 2013

6. Aveillant Ltd., http://www.aveillant.com/news/derbys-windturbines-see-development-world-first-radar-technology/, Accessed 6/3/15.

7. B. Gallardo-Hernando, F. Ṕerez-Martınez, and F. AguadoEncabo, Detection and mitigation of wind turbine clutter in cband meteorological radar, IET Radar Sonar \& Navigation, 4(4):520 -527, August 2010.

8. T.E. Derham, S. Doughty, K. Woodbridge, C.J. Baker, 'Design and evaluation of a low-cost multistatic netted radar system', IET Radar Sonar \& Navigation, 1, (5), pp.362-368, 2007. 